\title{
DIGITAL BEAM-FORMING RECONFIGURABLE RADAR SYSTEM DEMONSTRATOR
}

\author{
A. Patyuchenko, T. Rommel, P. Laskowski, M. Younis, G. Krieger \\ German Aerospace Center (DLR), Microwaves and Radar Institute \\ Muenchner Strasse 20, 82234 Wessling, Germany
}

\begin{abstract}
Synthetic Aperture Radar (SAR) based on the Digital BeamForming (DBF) concept belongs to the family of Multi-Modal Radar Systems (MMRS) offering higher operational flexibility and improved performance compared to conventional radar systems using analog beam steering. DBF SAR, in particular, overcomes the fundamental limitation of classical SAR systems and can deliver high resolution and simultaneously wide swath images. The purpose of this paper is to present a laboratory prototype of such a MMRS architecture - a reconfigurable radar demonstrator based on the DBF architecture. A hardware configuration of the radar as well as advanced concepts to be experimentally verified using it are considered and discussed.
\end{abstract}

Index Terms - digital beam-forming, radar, synthetic aperture radar, reflector, MIMO

\section{INTRODUCTION}

Next generation spaceborne Synthetic Aperture Radar (SAR) has to meet higher requirements for spatial and radiometric resolution, as well as coverage. This is conditioned by the success of the current SAR systems promoting the evolution of SAR and boosting the performance requirements for future systems. High resolution/wide swath performance tendency is recognized nearly independently of the application area which can be seen from several study programs initiated by space agencies and dedicated to future SAR systems. A review of several ongoing studies shows that a promising candidate for the next generation SAR is a multi-channel radar utilizing digital beam-forming (DBF) techniques [1], [2] which allows to overcome the fundamental high resolution/wide swath limitations of the conventional SAR systems [3].

A prototype of the next generation Multi-Modal Radar System (MMRS) - a reconfigurable DBF radar demonstrator - is described in this paper. The purpose of this paper is to present the MMRS prototype system, consider its hardware configuration, and introduce advanced concepts to be experimentally verified with its help.

The paper starts with an introduction of the MMRS demonstrator design which is followed by a description of

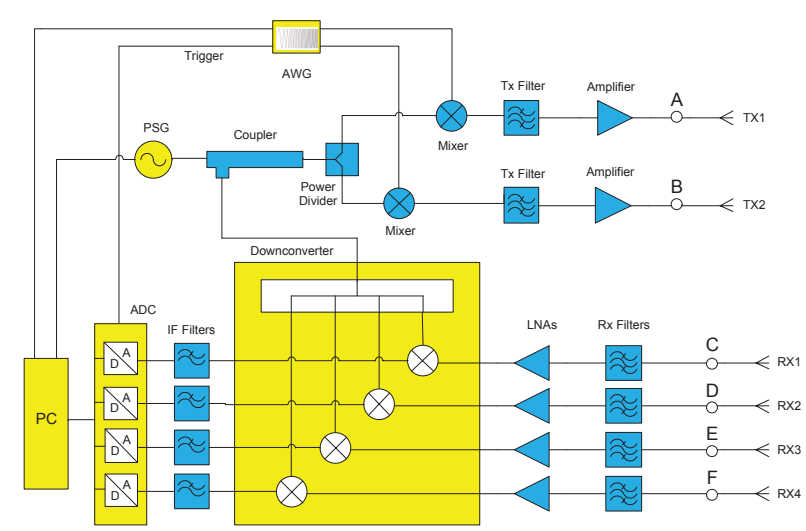

Fig. 1. Block diagram of the Digital Beam-Forming (DBF) system radar demonstrator.

its operational hardware and a calibration block configurations. Afterwards, concepts to be demonstrated using the radar are considered. The paper concludes with a summary.

\section{DIGITAL BEAM-FORMING DEMONSTRATOR}

An architecture of a modular reconfigurable multi-channel DBF radar demonstrator operating at multiple frequency bands is depicted in Figure 1. The system is based on the cPCI form-factor and configured to X-band operation using two transmit and four receive channels. This is the basic configuration intended to be used for first functionality tests, while the demonstrator will later be extended to more than eight independent receive channels and at least four transmit channels.

The desired complex chirp signal is synthesized using an Arbitrary Waveform Generator (AWG) and up-converted to the X-Band using an RF mixer and an analog signal generator (PSG). The up-converted RF signal is filtered using a bandpass filter, amplified and transmitted. At the receiver part the echo signals are independently received by each channel, amplified using Low Noise Amplifiers (LNAs), down-converted to the Intermediate Frequency (IF) band, and digitized. Data acquisition at each receive channel allows a posteriori com- 


\begin{tabular}{|l|l|}
\hline Parameter & Value \\
\hline operational frequency & $9.95 \mathrm{GHz}$ \\
max. output power & $33 \mathrm{dBm}$ \\
max. RF bandwidth & $500 \mathrm{MHz}$ \\
max. IF bandwidth & $300 \mathrm{MHz}$ \\
sampling rate & $2 \mathrm{GS} / \mathrm{s}$ \\
resolution & $10 \mathrm{bit}$ \\
max. data rate & $400 \mathrm{MB} / \mathrm{s}$ \\
\hline
\end{tabular}

Table 1. DBF hardware parameters

bination of the recorded signals to form multiple beams with adaptive shapes. The additional information about the direction of the scattered radar echoes can then be used to suppress spatially ambiguous signal returns, increase the receiving antenna gain, suppress spatially localized interferences, and gain additional information about the dynamic behaviour of the scatterers and their surroundings. The main parameters of the DBF demonstrator are summarized in Table 1.

The radar system end-to-end calibration block is schematically depicted in Figure 2. This functional block, implemented in hardware, is integrated into a compact box embedded into the radar demonstrator enabling an easy end-to-end system calibration by a simple electronic switching of signal paths without any hardware disassembling. The integration of the calibration block into the demonstrator is done by connecting the outputs of the DBF radar labeled in Figure 1 by letters A-F with the corresponding inputs of the calibration

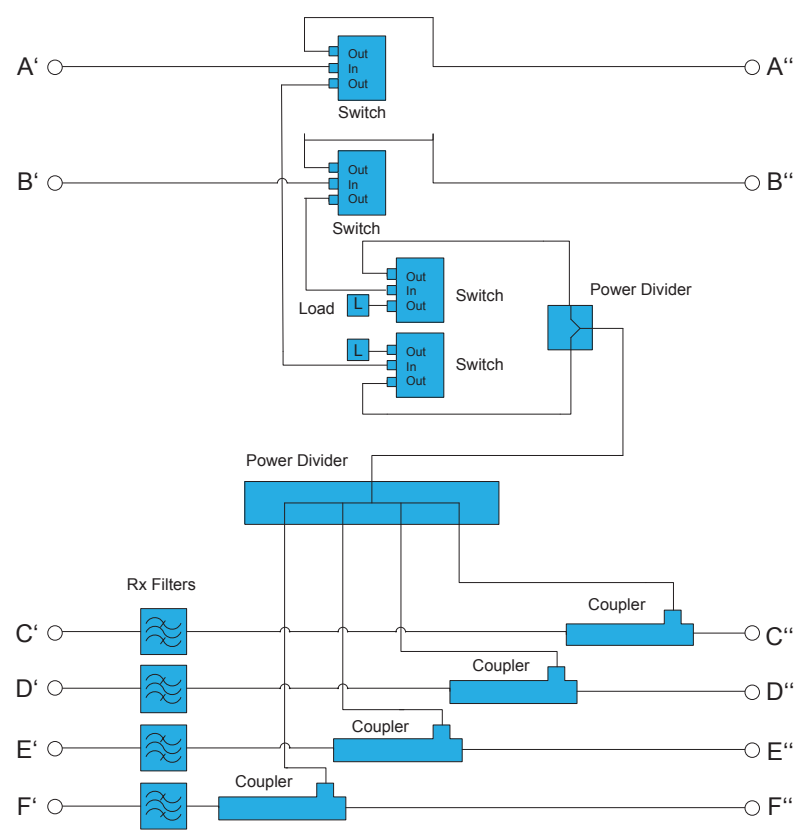

Fig. 2. Schematic representation of the calibration network.

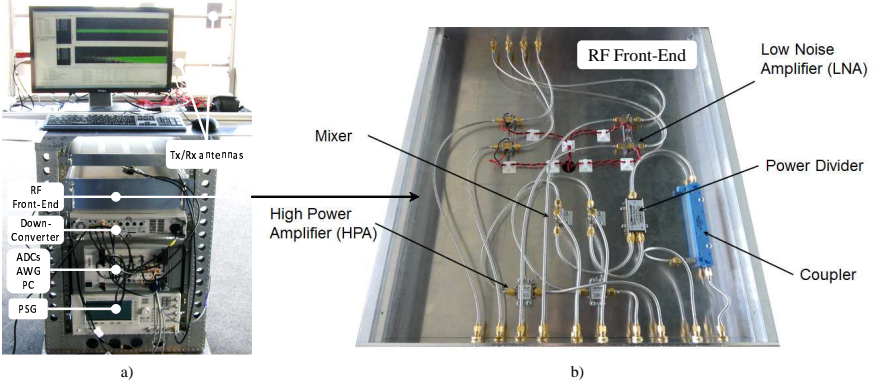

Fig. 3. DBF radar demonstrator (a) and the RF front-end block (b).

box labeled in Figure 2 by letters A'-F'. The transmit and receive antennas are in their turn connected to the outputs of the calibration box depicted in Figure 2 by letters A"-F".

This calibration block allows us to treat the entire radar measurement system with all its components and cabling between them as a single entity. With its help it is possible to remove the errors introduced from cabling between the components, wiring in the instrumentation front-end, and inaccuracies due to a non-linear behaviour of the individual system elements and antennas themselves. In addition to that the endto-end calibration block allows us to compensate channel synchronization errors and certain instrumentation components errors, such as offset errors.

An assembled DBF radar prototype and its RF front-end block are depicted in Figure 3 (a) and (b), respectively. The main objective of the demonstrator is the development and verification of innovative concepts and advanced operational modes for the future spaceborne imaging systems [4] as well as the research of their functional capabilities and limitations. These concepts and imaging techniques have the potential to significantly enhance the performance of future systems compared to the state-of-the-art SAR sensors like TerraSARX/TanDEM-X, Radarsat-2 or Sentinel-1.

\section{INNOVATIVE CONCEPTS}

The first two innovative MMRS configurations, encompassing diverse research questions, which have to be realized and thoroughly studied using the reconfigurable MMRS demonstrator are briefly introduced in the following.

\subsection{Reflector-based DBF SAR}

The DBF SAR system using a reflector antenna is a novel concept offering better performance and a lower level of complexity compared to the DBF radars using planar antennas. Spaceborne systems based on this concept are being currently studied and have already been discussed in a number of publications including [5], [6]. In order to demonstrate the operation of the reflector based DBF radar and verify theoret- 

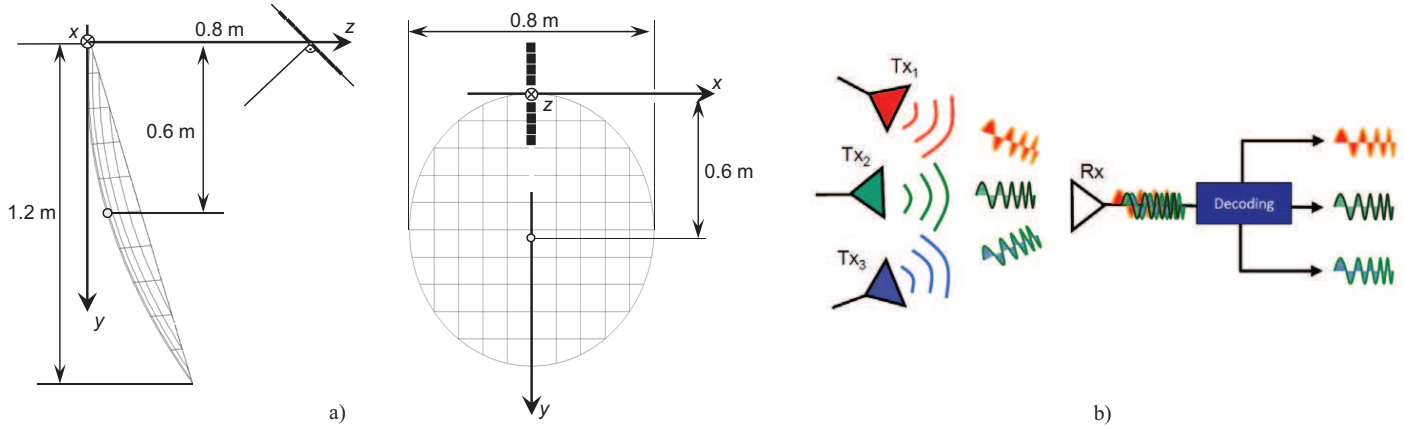

Fig. 4. A multi-beam antenna with a focal plane array for a reflector-based DBF SAR (a) and a MIMO radar concept (b)

ically obtained results, the reflector based configuration of the demonstrator was designed. For this, an elliptical antenna with a size of $1.2 \times 0.8 \mathrm{~m}$, a focal length of $0.8 \mathrm{~m}$ and an offset clearance of $0.6 \mathrm{~m}$ in major axis was chosen. The feed system consists of 8 horn antennas offering an optimum illumination of the antenna surface. Its architecture is highly flexible allowing a change of the inter-element spacing between feeds as well as of the position of the whole system relative to the antenna. The reflector antenna design is schematically shown in Figure 4 (a).

Using such a configuration it will be possible to test various operational options of the reflector based DBF SAR among which are advanced operational modes in bi- and mono- static configurations, inverse DBF SAR, and the use of the Conjugate Field Matching principle to improve antenna patterns [7]. In addition, certain effects observed in such systems can also be studied, among others, the impact of the reflector surface shape and possible deformations on the DBF performance [8], frequency dependent effects [6] and the influence of specific antenna parameters on DBF radar characteristics.

\subsection{Multiple-Input Multiple-Output radar}

Another advanced concept to be experimentally investigated and verified is the Multiple-Input Multiple-Output (MIMO) DBF radar [9]. The MIMO radar can make use of orthogonal waveforms obtained using, e.g., the novel Orthogonal Frequency Division Multiplexing (OFDM) scheme [10]. These orthogonal waveforms are simultaneously transmitted using multiple transmit channels while their echoes are later acquired using multiple digital receive channels (Figure 4 (b)). Thus, the MIMO radar incorporates advanced features in addition to High-Resolution Wide-Swath (HRWS) SAR imaging [11] offering improved imaging performance, and making it possible to acquire more information about targets. The purpose of the MMRS demonstrator is to experimentally verify, e.g., the use of the OFDM chirp scheme [9] as well as to demonstrate the high potential of MIMO imaging systems for future spaceborne remote sensing applications.

\section{CONCLUSION}

A modular reconfigurable DBF radar demonstrator was considered in this paper. Its operational hardware and calibration block were presented and discussed. Advanced concepts to be studied and experimentally verified using this demonstrator were considered.

The configuration of the radar demonstrator can be extended by increasing the number of receive and transmit channels. The use of several transmit channels with orthogonal signals (MIMO concept) would enhance the capabilities of the radar by the possibility to employ innovative operational modes considerably improving the performance and flexibility of the system. The use of a reflector antenna in a combination with the DBF radar would in its turn enable a realisation of advanced concepts as well as allow the verification of performance improvement methods.

The radar demonstrator presented in the paper is the prototype of the next generation Multi-Modal Radar Systems (MMRS) which will be able to satisfy the stringent requirements of future spaceborne remote sensing radars to achieve high resolution and wide coverage of the imaged area.

The results of the development of the advanced DBF radar demonstrator presented in this paper are a very first step towards the future spaceborne Multi-Modal Radar Systems, such as MIMO radars using orthogonal waveforms and reflector-based DBF SAR. Innovative imaging techniques and technologies employed in these systems have the potential to considerably enhance the imaging performance of future spaceborne radars compared to state-of-the-art SAR sensors. 


\section{REFERENCES}

[1] C. Schaefer, M. Younis, and M. Ludwig, "Advanced SAR instrument based on digital beam forming," in Advanced RF Sensors and Remote Sensing Instruments, Noordwijk, Netherlands, November 2009.

[2] C. Fischer, C. Schaefer, and C. Heer, "Technology development for the HRWS (high resolution wide swath) SAR," in International Radar Symposium IRS'07, September 2007.

[3] A. Currie and M. A. Brown, "Wide-swath SAR," IEE Proceedings $F$ - Radar and Signal Processing, vol. 139, pp. 122-135, 1992.

[4] G. Krieger, M. Younis, N. Gebert, S. Huber, F. Bordoni, A. Patyuchenko, and A. Moreira, "Advanced digital beamforming concepts for high performance synthetic aperture radar (SAR) imaging," in Advanced RF Sensors and Remote Sensing Instruments (ARSI), Noordwijk, Netherlands, November 2009.

[5] M. Younis, S. Huber, A. Patyuchenko, F. Bordoni, and G. Krieger, "Performance comparison of reflector- and planar-antenna based digital beam-forming SAR," International Journal of Antennas and Propagation, vol. 2009, pp. 13 pages, 2009.

[6] A. Patyuchenko, M. Younis, S. Huber, and G. Krieger, "Performance optimization of the reflector antenna for the digital beam-forming sar system," in ESA Workshop on Advanced RF Sensors and Remote Sensing Instruments, Noordwijk, Netherlands, November 2009.

[7] S. Huber, M. Younis, A. Patyuchenko, and G. Krieger, "Digital beam forming techniques for spaceborne reflector sar systems," in European Conference on Synthetic Aperture Radar (EUSAR), Aachen, Germany, June 2010.

[8] A. Patyuchenko, M. Younis, S. Huber, and G. Krieger, "Reflector-based digital beam-forming SAR with improved azimuth performance," in ESA Antenna Workshop, Noordwijk, Netherlands, October 2010.

[9] Junghyo Kim, Multiple-Input Multiple-Output Synthetic Aperture Radar (SAR) for Multimodal Operation, Ph.D. thesis, Karlsruhe Institute of Technology (KIT), 2011.

[10] G. Krieger, N. Gebert, and A. Moreira, "Multidimensional waveform encoding: A new digital beamforming technique for synthetic aperture radar remote sensing," IEEE Transactions on Geoscience and Remote Sensing, vol. 46, no. 1, pp. 31-46, 2008.
[11] M. Suess, B. Grafmueller, and R. Zahn, "A novel high resolution, wide swath SAR system," in IEEE International Geoscience and Remote Sensing Symposium (IGARSS), Sydney, Australia, 2001. 